\title{
Recent trends in Mobile Communication Systems
}

\author{
Sang-Yeob Oh • Supratip Ghose • Hye-Jung Jang • \\ Kyungyong Chung
}

Published online: 25 March 2014

(C) Springer-Verlag France 2014

With recent expansions of IT convergence equipment, more and more mobile communications-related devices are used in many fields, and mobile communications_-based projects are underway in various venues, such as u-cities, u-ports, u-health, and u-traffic. In particular, owing to increasing demand for personal services due to the rapid increase in the elderly population and rapid development of convergence technology, the increased competition among mobile communications institutions, etc., is shifting towards consumeroriented services, giving priority to the expectations and desires of consumers when delivering ubiquitous services. As a result, the use of mobile communications technology is increasing in convergence-related fields. Mobile communications systems for convergence environments pose many research challenges. Among these is processing overhead in service environments [13-18]. Therefore, novel techniques, architectures, algorithms, and experiences regarding multimedia applications and industry services must be considered. Some of these research areas are listed below.

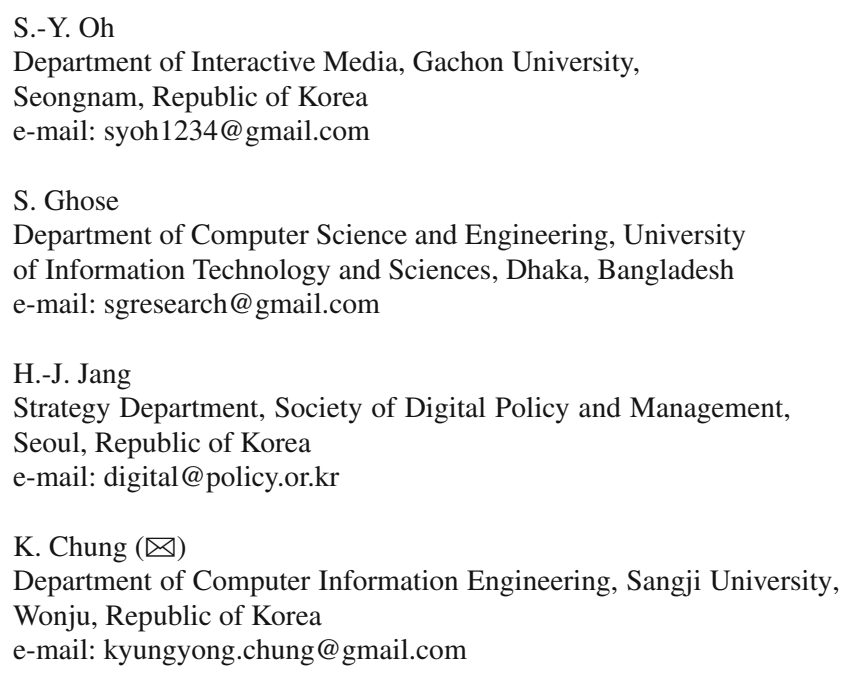

- Ubiquitous Computing for Convergence

- Software Infrastructure, Middleware and Frameworks for Convergence Information

- Mobile and Wearable Computing for Convergence Information

- Agent Technology in Convergence Information Systems

- Convergence Information Applications in u-Commerce, u-Learning, u-Health

- Two-way Authentication Protocols in Wireless Communications

- Innovative Authentication and Authorization of Mobile Users

- Device Identities and Authentication

- Mobility for Convergence

- Mobile Identity Management

- Enhanced Authentication Control Systems for Mobile and Wireless Systems

- Authentication in Mobile Networks

- Convergence Information Indexing, Searching, and Visualization

- Intelligent Information Extraction Algorithms

This special issue is devoted to one of the hottest topics in mobile communication systems, and its articles are expected to be cited widely in the areas of computer virology and hacking techniques.

Lee et al. [1] present Choquet integral-based product satisfaction inference taking into consideration subjective decision-making tendencies. They propose a product satisfaction inference method reflecting users' preferences based on such variables as price, discount rate, number of buyers, and purchase satisfaction, which are most influential in choosing and purchasing goods. For this purpose, the paper includes product satisfaction evaluation using a fuzzy set theory—based fuzzy-inference system and the 
fuzzy measure theory-based fuzzy integral. The outcome of the paper indicates that it is possible to recommend products by means of an analytical hierarchy process and fuzzy integrals in reference to objective product data. Sim and Baek [2] introduce a study of a scalable informationmatching system based on web service information. This study proposes a decision process in terms of a web service function for users in real-life situations using conventional methods and services. Scalable information is used to subsequently propose a service selection broker so that an optimal web service can be selected when users seek a web service. The service selection process proposed in this thesis simplifies the model by utilizing Web Services Description Language (WSDL) and Universal Description, Discovery and Integration for the existing service search model without dynamically applying the web service level contract or web service policy statement during execution time when clients' requests for services do not pass beyond the service search model used in the web service search method.

Kim et al. [3] present indoor location tracking based on mobile radio frequency identification (RFID) for a smart exhibition service. They propose the scenario and architecture of an indoor location tracking service for things or space in an exhibition environment based on mobile RFID. The proposed indoor location tracking system helps to find the locations of users, things and spaces to provide effective exhibition services. The RFID tags are identified as locations of points that people pass, and they obtain spatial data from the tags using mobile RFID readers, a web server and a database server. The authors provide an intelligent exhibition service by presenting a new direction using the proposed system. Kim et al. [4] introduce a heuristic estimation of network capacity and spectrum requirements. An efficient estimation method for network capacity and spectrum requirements is proposed based on transform free analysis for waiting time distribution in an $\mathrm{M} / \mathrm{G} / 1$ priority queue. The mean waiting time of an M/G/1 non-preemptive priority queue is analyzed by the heuristic method mainly composed of the first and second moments of transmission time for each type of packet. The proposed heuristic approach could simplify the estimation process for the required capacity and spectrum requirements. It can also be applied to the analysis of an optional situation in a network, which permits vacation time for maintenance or warm up.

Hong et al. [5] present implementation of a smart pulsimeter. They propose an algorithm to analyze weak pulse wave symptoms and strong pulse wave symptoms. It uses a portable pulsimeter that includes a Hall effect sensor. In this research, it was suggested that a rapid pulse and a weak pulse could be discerned if we would use a portable pulsimeter that is designed on the basis of Hall sensor capabilities to clear problems like those mentioned above. Hav- ing analyzed patients with a rapid pulse and those with a weak pulse by statistical methods, the researchers prove that $\mathrm{N}$ time (notch point time) and $\mathrm{S}$ Amp (rise waveform size) mean values are significantly different, based on a $95 \%$ confidence interval. Lim and Kim [6] introduce modeling discovery and removal of security vulnerabilities in a software system using priority queuing models. This paper aims to model the discovery and removal of software vulnerabilities based on queuing theory. The probabilistic characteristics of the arrival and service processes are the core elements of queuing theory. The proposed method exploits priority queuing models with batch arrivals. Specifically, $\mathrm{Mx} / \mathrm{G} / 1$ queuing models with batch arrivals and different classes are introduced. To make the model more valuable, other unmodeled phenomenon should also be considered. Variation of discovery rates over time and versions can also be taken into account.

Choi et al. [7] present an adjusting context-aware RFID for a health screening center (HSC). They propose an example that provides information to examinees and the HSC through a health-screening workflow with RFIDs. When the examinee has arrived and has finished the test, the signal notifies the lab's PC and is then transferred to the server. After receiving a signal, the workflow system launches a simulation by analyzing pre-defined routes, the number of people waiting and average test times. This study applied context-awareness to the HSC's workflow. After analyzing related technologies and methods, the authors found this a suitable solution for HSC workflow. This research suggests that HSCs should implement a context-aware RFID system. Kim et al. [8] discuss a study on the factors that affect the adoption of a smart water grid, aiming to review previous studies to identify factors that influence adoption of a smart water grid and to analyze the importance and priority of the factors affecting smart water grid adoption, thereby presenting successful adoption measures for smart water grids in Korea. This study established a research model from influencing factors related to the adoption of new IT technologies identified through literature reviews of previous studies based on the technology-organization-environment framework. This study of the factors that affect the adoption of a smart water grid will be the basis for effective adoption and activation of this next generation of water management system.

Ahn et al. [9] present a low complexity image stitching using advanced feature matching in mobile environments. They propose a method that extracts the minutiae in each image and looks for the corresponding matching points in overlapped parts of the image. To find these points, the algorithm matches feature points via bidirectional feature matching to reduce computational complexity, rather than with the conventional single matching method. They also eliminate unnecessary matching points that are not clustered to 
reduce processing time, and the transformation matrix to merge corresponding images is generated by selected matching points. Jeong [10] present a loop-splitting method for single loops with non-uniform dependences. They propose a generalized and optimal method to make the iteration space of a loop into partitions with variable sizes. They developed an improved loop-splitting method for maximizing parallelism of single loops with non-constant dependence distances. By using the iteration and distance for the source of the first dependence, and with their defined theorems, they present generalized and optimal algorithms for single loops with non-uniform dependences. Their algorithm generalizes how to transform general single loops with flow and antidependences into parallel loops. In comparison with some previous splitting methods, the proposed methods provide much better speedup and extract more parallelism than other methods.

Choi and Han [11] introduce a study on the service quality of SME management consulting affecting perceived management performance in mobile communications environments. For the purpose of this study, the service quality of a consulting firm was chosen to be the preceding factor that has an effect on the improvement of performance based on a pilot study. Parameters were set to differentiate this from other studies, including whether it has been supported by the state, what the consulting area is like, and whether a consultant has relevant certification. A theoretical research model was established based on a pilot study, questionnaire items were adjusted and redesigned to reflect the research subject, and actual proofs were analyzed with a view to determine whether service quality of management consulting has an effect on better performance. Kim and Park [12] discuss legal limits of search and seizure for digital forensics in Korea. The purpose of this research was to examine concepts and types of cyber-crime, and to trace trends in the Republic of Korea over recent years. If made accessible, information stored in private computers has the potential to serve as decisive evidence in many criminal prosecutions. However this partial code does not explicitly define the admissibility of digital evidence and acceptable methods of examining it. Hence, one of the goals of this research was to identify problems pertaining to the law and suggest ways to improve it.

This fine collection of papers was accumulated by fruitful collaboration. We gratefully acknowledge and express our heartfelt appreciation to all the authors for their excellent contributions to this special issue. We would also like to thank all the members of the SDPM, ICDPM Program Committee and anonymous reviewers for their help in identifying novel papers and for their careful reading of earlier drafts to select 12 high-quality papers out of 25 papers submitted-a $48 \%$ acceptance rate. Furthermore, we would like to thank Professor Eric Filiol, editor-in-chief of the International Journal of
Computer Virology and Hacking Techniques, for his valuable remarks and help throughout the publication process of this special issue.

\section{References}

1. Kwon, I., Lee, S.: Choquet integral-based product satisfaction inference in consideration of subjective decision-making tendencies. J. Comput. Virol. Hacking Tech. (2013). doi:10.1007/ s11416-013-0199-y

2. Sim, S., Baek, S.: A study on scalable information matching system based on web service information. J. Comput. Virol. Hacking Tech. (2014). doi:10.1007/s11416-014-0204-0

3. Kim, S., Park, H., Bang, H., Kim, D.: An indoor location tracking based on mobile RFID for smart exhibition service. J. Comput. Virol. Hacking Tech. (2014). doi:10.1007/s11416-014-0200-4

4. Kim, T., Park, H., Kim, B.: Heuristic estimation of network capacity and spectrum requirement. J. Comput. Virol. Hacking Tech. (2013). doi:10.1007/s11416-013-0198-z

5. Hong, Y., Kim, M.K., Kim, G.H.: Implementation of a web-based smart electronic needle system. J. Comput. Virol. Hacking Tech. (2014). doi:10.1007/s11416-014-0201-3

6. Lim, D., Kim, T.: Modeling discovery and removal of security vulnerabilities in software system using priority queueing models. J. Comput. Virol. Hacking Tech. (2014). doi:10.1007/ s11416-014-0205-Z

7. Choi, Y., Lee, G., Ra, J., Han, W.M.: Adjusting context-aware RFID in health screening center. J. Comput. Virol. Hacking Tech. (2014). doi:10.1007/s11416-014-0211-1

8. Kim, D., Park, K., Choi, G., Min, K.: A study on the factors that affect the adoption of smart water grid. J. Comput. Virol. Hacking Tech. (2014). doi:10.1007/s11416-014-0206-y

9. Ahn, H., Lee, Y., Lee, J., Cho, H.: A low complexity image stitching using advanced feature matching in mobile environments. J. Comput. Virol. Hacking Tech. (2014). doi:10.1007/s11416-014-0207-x

10. Jeong, S.J.: A loop splitting method for single loops with nonuniform dependences. J. Comput. Virol. Hacking Tech. (2014). doi:10.1007/s11416-014-0208-9

11. Choi, M., Han, K.: A study on the service quality of SMEs management consulting affecting the perceived management performance in mobile communication environments. J. Comput. Virol. Hacking Tech. (2014). doi:10.1007/s11416-014-0209-8

12. Kim, S.G., Park, J.S.: Legal limits of search and seizure for digital forensic in Korea. J. Comput. Virol. Hacking Tech. (2014). doi:10. 1007/s11416-014-0210-2

13. Ha, O.K., Song, Y.S., Chung, K.Y., Lee, K.D., Park, D.: Relation model describing the effects of introducing RFID in the supply chain: evidence from the food and beverage industry in South Korea. Pers. Ubiquitous Comput. 18(3), 553-561 (2014)

14. Kim, S.H., Chung, K.Y.: 3D simulator for stability analysis of finite slope causing plane activity. Multimed. Tools Appl. 68(2), 455-463 (2014)

15. Boutaba, R., Chung, K., Gen, M.: Recent trends in interactive multimedia computing for industry. Clust. Comput. (2014). doi:10. 1007/s10586-014-0349-0

16. Jung, E.Y., Kim, J.H., Chung, K.Y., Park, D.K.: Home health gateway based healthcare services through U-health platform. Wirel. Pers. Commun. 73(2), 207-218 (2013)

17. Oh, S.Y., Chung, K.Y.: Target speech feature extraction using nonparametric correlation coefficient. Clust. Comput. (2013). doi:10. 1007/s10586-013-0284-5

18. Kim, J.H., Ryu, J.K.: Recent trends on high-performance computing and security. Clust. Comput. 16(2), 207-208 (2013) 


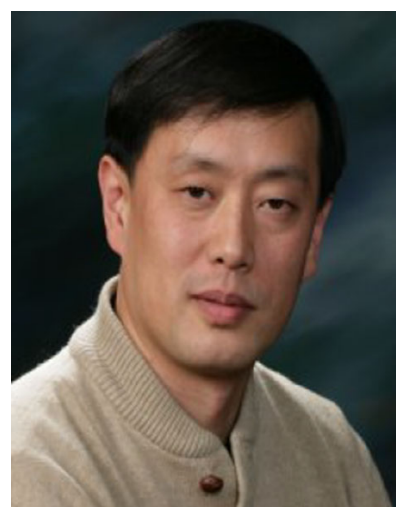

Sang-Yeob Oh received a B.S. in Computer Science from Kyungwon University in 1989 and an M.S. and Ph.D. from Kwangwoon University, Korea, in 1991 and 1999, respectively, from the Department of Computer Science. He is currently a professor in the Division of Computer Media, Gachon University, Korea. His research interests include Speech Recognition, Vehicle Safety Communications, and HCI. He has edited 27 computer science books. He serves as Executive Editing Director for the International Conference on Digital Policy Management, and as Executive Editing Director on the Steering Committees for the International Conference on Convergence Technology. He is also an editorial member of the International Journal of Wireless Personal Communications, among others.

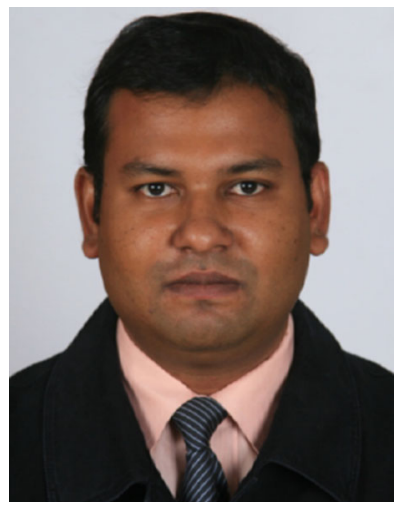

Supratip Ghose graduated from the Department of Applied Physics and Electronics, University of Rajshahi, with a BSc and an M.Sc. in 1995 and 1998, respectively, and received his Ph.D. in Information Engineering from the Department of Computer Science and Information Engineering, Inha University, South Korea, in 2007. Dr. Ghose began his academic career as an Adjunct Lecturer at Inha University. After obtaining his $\mathrm{PhD}$, he served as an Assistant Professor in the Department of Computer Science and Engineering in UIC-BNU-HKBU in Zhuhai, China, at the University of Saint Joseph, Macau, China, and at Brac University in Dhaka, Bangladesh. He is currently serving as an Associate Professor in the Department of Computer Science and Engineering, University of Information Technology and Sciences, Dhaka, Bangladesh. His research interests include Data Mining, Collaborative Filtering, Sentiment Analysis in Social Webs, IR Frameworks, and Activity Recognition in Smartphones.

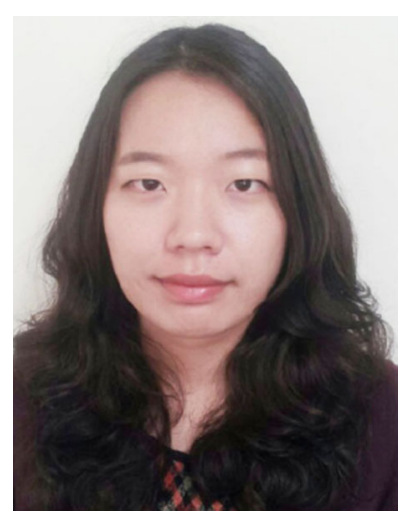

Hye-Jung Jang is the executive secretary of Society of Digital Policy and Management, Korea. She supervised and managed conferences including International Conference on Digital Policy and Management (ICDPM), International Conference Convergence Technology (ICCT), and International Conference for Small and Medium Business (ICSMB). Her research topics are Contents Design, HCI, Intelligent System, IT Convergence, Ubiquitous Computing, Serious Game, Game Planning, and Recommendation. Furthermore, she has controlled special issues of prestigious international journals. Also, she is editorial board members of several International Journals.

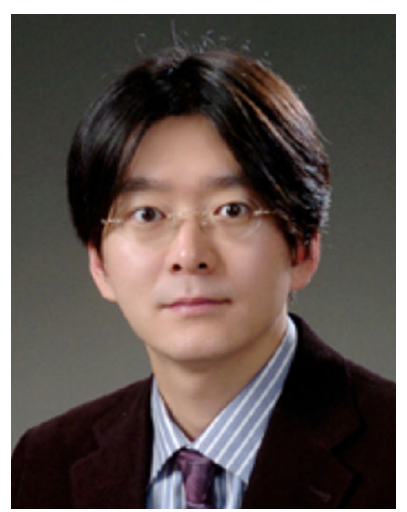

Kyungyong Chung is a Professor in the Department of Computer Information Engineering at Sangji University, Korea. $\mathrm{He}$ worked for the Software Technology Leading Department Korea IT Industry Promotion Agency (KIPA) in 2005. He received a $\mathrm{BS}$, an $\mathrm{MS}$, and a $\mathrm{PhD}$ in Computer and Information Engineering from Inha University in 2000, 2002, and 2005, respectively. His research topics include Data Mining, Telemedicine, Knowledge-based Decision Support Systems, Intelligent Systems, Convergence, HCI, and Recommendation Systems. He served as General Co-Chair for the International Conference on Information Science and Application 2013, as General Co-Chair of 2nd International Conference on IT Convergence and Security 2012, as Executive Editing Director on the Steering Committees for the International Conference on Convergence Technology, and on the Steering Committees for the International Conference on Digital Policy and Management. 